\title{
Compact, Low Power and Low Threshold Electrically Pumped Micro Disc Lasers for 20Gb/s Non Return to Zero All Optical Wavelength Conversion
}

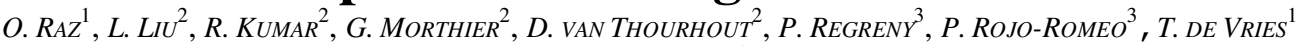 \\ ,H.J.S. DORREN ${ }^{1}$ \\ (1) Eindhoven University of Technology, Den Dolech 2, 5600MB, Eindhoven, The Netherlands (o.raz@tue.nl) \\ ${ }^{(2)}$ INTEC, Ghent University-IMEC, St-Pietersnieuwstraat 41, 9000 Ghent, Belgium \\ ${ }^{(3)}$ Institut des Nanotechnologies de Lyon INL-UMR5270, CNRS, Ecole Centrale de Lyon, Ecully F-69134, France
}

\begin{abstract}
Using a $7.5 \mu \mathrm{m}$ wide InP Micro-Disc-Laser, with a very low $\sim 100 \mu \mathrm{A}$ threshold current, heterogeneously integrated on top of Silicon on Insulator substrate, all optical NRZ wavelength conversion at speeds up to $20 \mathrm{~Gb} / \mathrm{s}$ is demonstrated
\end{abstract}

(C)2009 Optical Society of America

OCIS codes: (130.7405) Wavelength conversion devices; (230.1150) All-optical devices

\section{Introduction}

Micro Disc Lasers (MDLs) have attracted a lot of research interest as they can fulfil many optical functions in a very efficient way. One such attractive function is wavelength conversion which is a key functionality in photonic networks [1]. At present in most applications, wavelength conversion is implemented using nonlinear phenomena like Four Wave Mixing (FWM), Cross Gain or Cross Phase Modulations (XGM, XPM) in either nonlinear fibers, semiconductor optical amplifiers based structures or silicon waveguides [2-5]. These approaches have in common that they use either high electrical or optical powers and in some case employ bulky nonlinear media. Moreover, high-speed wavelength conversion requires return-to-zero (RZ) data formats [5] whereas wavelength conversion for non-return-to-zero (NRZ) On-Off Keying (OOK) signal is most desirable. A major step forward would be an all-optical wavelength converter that occupies only a few square $\mu \mathrm{m}^{2}$ chip space and dissipates only a few $\mathrm{mW}$ of electrical and optical power, while still having the ability to operate at high speeds, using NRZ data formats. This enables the integration of hundreds or even thousands of wavelength convertors on a single chip. A promising candidate for such wavelength converters are MDLs as their typical foot print and/or power consumption are $10^{1}-10^{3}$ times smaller compared to an InP SOA or a silicon waveguide [2-5]. Previously reported operation of such structures [6] for wavelength conversion has yielded a BER of $\sim 10^{-4}$ at a bit rate of $2.5 \mathrm{~Gb} / \mathrm{s}$.

In this paper we present results of a new generation of InP MDLs bonded on top of Silicon-on-Insulator (SOI). We demonstrate error-free wavelength conversion at 2.5 and $10 \mathrm{~Gb} / \mathrm{s}$ with minimal to medium penalties respectively and at $20 \mathrm{~Gb} / \mathrm{s}$ BER below $10^{-3}$, which is the minimal limit for FEC error free operations, can be obtained.

\section{Advances in MDL Performance}

Previous generations of bonded InP membrane MDLs gave several $\mu \mathrm{Ws}$ of fiber coupled output power and had relatively high threshold currents with noticeable gain deterioration due to device heating up for currents exceeding five to six times the threshold current [6] (thermal roll-off). Major changes in the design, fabrication and InP epitaxial growth of these devices have been implemented yielding MDLs with dramatically enhanced performance. A detailed description of all improvements can be found in [7], but for clarity, we briefly mention the changes here. The tunnel junction layer, previously residing between the active layer and the SOI substrate, leading to absorption losses has been relocated to above the active layer. Additionally the entire layer stack was thinned to lower losses due to recombination at the disc's edge. The etching of the disc has been carried out by Inductive Coupled Plasma RIE (ICP-RIE) yielding much lower side wall roughness (see Fig. 1).
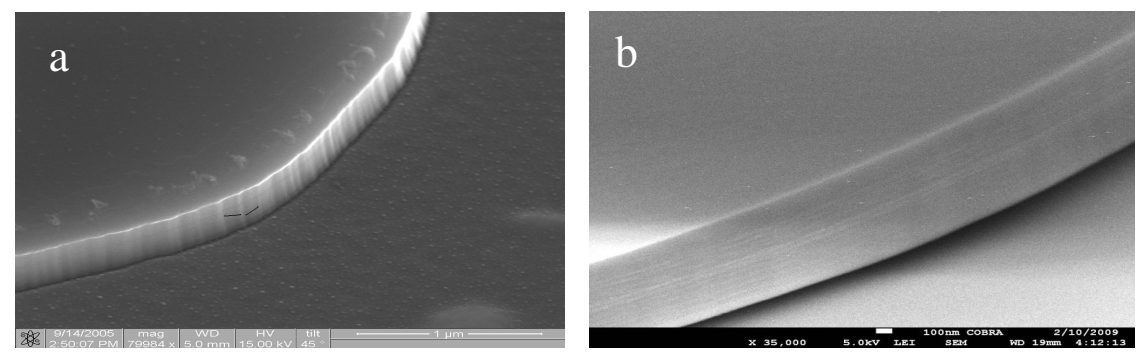

Fig. 1. SEM images of MDLs side walls (a) RIE (b) ICP-RIE 
The current injection was improved by shaping the bottom electrode to follow the contour of the disc. Finally, larger metal contacts on top of the disc contact pad have been put in place. The outcome of these changes is a dramatic improvement in device performance compared to the device used in ref 6 ; the fiber coupled powers have shot up with a factor 10 to 20 (to $20 \mu \mathrm{W}$ ) and the lasing threshold current has dropped 5 fold (to $100 \mu \mathrm{A}$ ). Typical I-L curves as well as representing spectra for an MDL with an injection current are shown in Fig. 2.

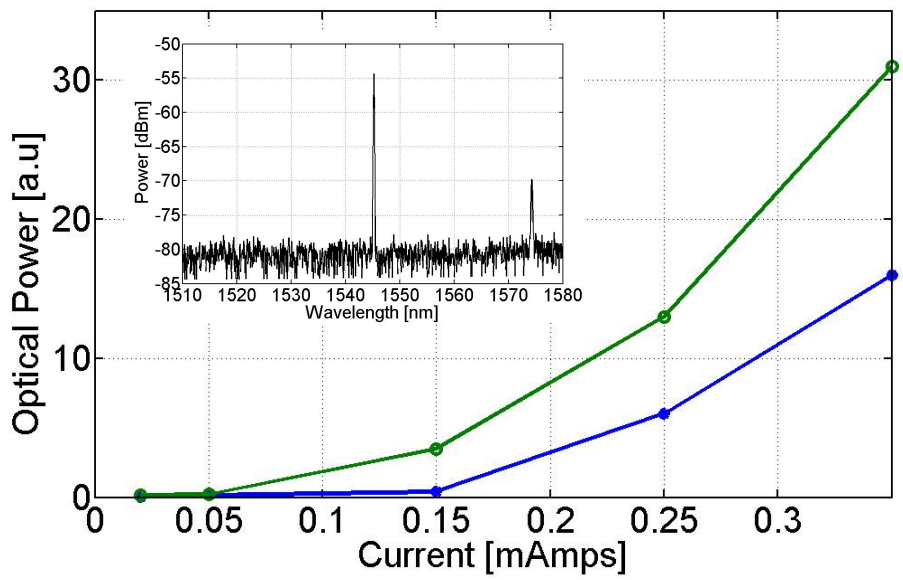

Fig. 2. I-L curves for low threshold MDLs (optical spectra of one MDL for a current of 150 microAmps in inset)

This implies that the laser can be driven at 20 to 30 times the threshold current which has important implications for the possible modulation speed [8].

\section{Experimental Set-up}

The experimental set-up is shown in Fig. 3.

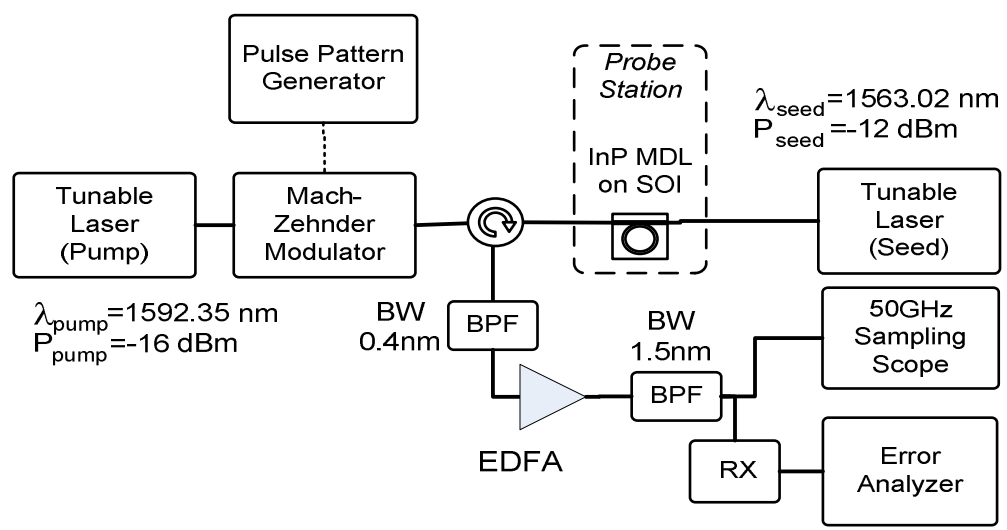

Fig. 3. Experimental set up

The pump signal is a tunable laser source tuned to the non-dominant lasing mode of the MDL and modulated by a PRBS generator at 2.5,10 and 20Gb/s using an MZM. From the other side of the disc a 2nd laser (seed) is tuned to the dominant lasing mode under free running operation (see further details on necessity of seed laser below). Power levels are assumed in the silicon waveguide based on power in the input to the fiber lens and assuming $8 \mathrm{~dB}$ coupling losses. The free running laser light at the circulator's output is filtered, amplified using an EDFA and filtered again to remove excessive ASE noise before detection at the appropriate receivers (APD for 2.5 $\mathrm{Gb} / \mathrm{s} ; 10 \mathrm{~Gb} / \mathrm{s}$, PIN for $20 \mathrm{~Gb} / \mathrm{s})$. The device was a $7.5 \mu \mathrm{m}$ diameter InP disk laser lithographically etched in a $580 \mathrm{~nm}$ thick membrane bonded on top of an SOI waveguide circuit. The disc was operated at $15^{\circ} \mathrm{C}$ and biased at $2.5 \mathrm{~mA}$ (3volt) to give $\sim 10 \mu \mathrm{W}$ of power in the fiber. Gratings at both ends of the silicon waveguides allowed for coupling of light to the chip.

\section{Results}

Fig. 4 shows the MDLs output spectra under three operation conditions. On the top, free running operation is shown, indicating $>20 \mathrm{~dB}$ side mode suppression. Once the pump laser is turned on, the free running laser power (middle frame) drops by $9 \mathrm{~dB}$ due to the limited recovery speed of the MDL, effectively inhibiting conversion. The solution is to introduce the seed laser [6], which speeds the MDL free running mode recovery, gaining back $6 \mathrm{~dB}$ to leave an expected $3 \mathrm{~dB}$ power gap between modulated and un-modulated spectra's (bottom frame). For different modulation speeds the reference performance of the receiver (Receiver Power vs. BER) were measured and compared to the wavelength converted signals. Curves for all measured modulation speeds are shown in Fig 
5. For the $2.5 \mathrm{~Gb} / \mathrm{s}$ and $10 \mathrm{~Gb} / \mathrm{s}$ signals the EDFA operated with low to moderate gains yielding $0.8 \mathrm{~dB}$ and 4.5 $\mathrm{dB}$ penalty respectively, mainly depending on available OSNR.

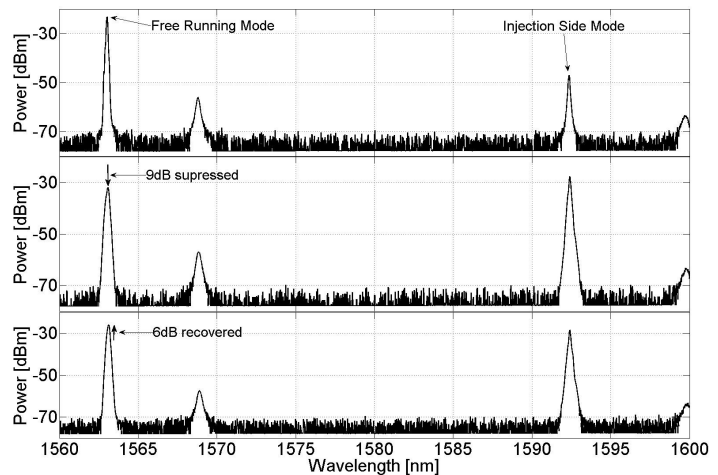

Fig. 4. Measured spectra of disc laser; free running (top), only with pump (middle), both pump and seed (bottom)
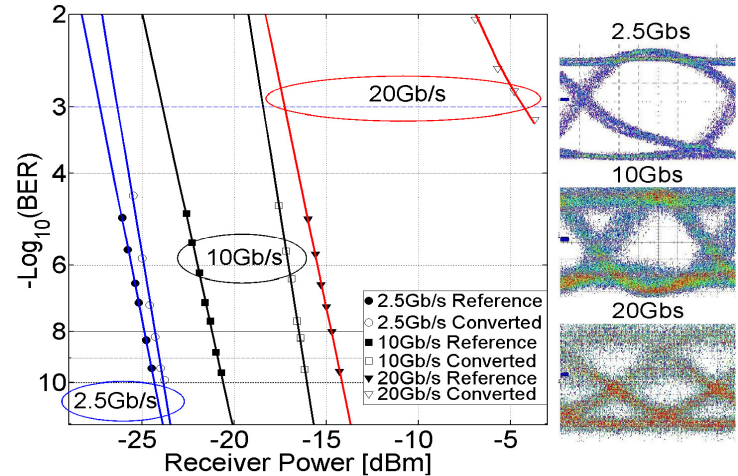

Fig. 5. Measured BER for MDL output for three different bit rates (eye patterns are given on the right)

For $20 \mathrm{~Gb} / \mathrm{s}$ rate, the receiver used had twice the required bandwidth (43Gb/s module), doubling the noise contribution, and much lower sensitivity forcing higher gain from the EDFA and further degrading the noise figure to leave a distinct error floor. Power was kept below $-3 \mathrm{dBm}$ to avoid damaging the receiver resulting in BER under $10^{-3}$ but no error free operation.

\section{NRZ Conversion - Figure Of Merit (FOM)}

As commented in the introduction criteria such as power consumption and area on the chip are detrimental in assessing the usability of any All-Optical Wavelength Conversion (AOWC). We therefore suggest using the following FOM:

$$
\text { FOM }=\frac{\text { Data Speed }[\mathrm{Gb} / \mathrm{s}]}{\text { Power }[\text { mWatt }] \cdot \text { Area }\left[\mu \mathrm{m}^{2}\right]}
$$

Table 1 compares the FOM for the four methods discussed in this paper.

\begin{tabular}{l|cc|cc}
\hline & InP-SOA [4]/[5+] & SOI [2] & SOI [3] & MDL \\
\hline Top speed [Gb/s] & $40 / 320^{+}$ & $2.5^{* \dagger}$ & $1^{*}$ & $10^{* * *}$ \\
Power $[\boldsymbol{m} \boldsymbol{W}]$ & $1000 / 1000^{+}$ & $200^{* *}$ & $200^{* *}$ & 10 \\
Area $\left[\boldsymbol{\mu m}^{2}\right]$ & $4000 / 2000^{+}$ & 300 & 80 & 40 \\
FOM & $\sim 1 \cdot 10^{-5} / 1.6 \cdot 10^{-4}$ & $\sim 5 \cdot 10^{-5}$ & $\sim 5 \cdot 10^{-5}$ & $2.5 \cdot 10^{-2}$ \\
\hline
\end{tabular}

Table 1. FOM comparison

* No BER values given;** Electrical power needed to drive SOA to boost optical power to the required levels - conservative estimate; *** Speed for the fastest error free operation; $\dagger$ based on $100 \mathrm{psec}$ response time; $+\mathrm{RZ}$ modulation format

\section{Discussion and Conclusions}

We have demonstrated AOWC with a FOM 2 orders of magnitude better than any previously reported integrable device, using very conservative estimates. The suggested MDL based AOWC is unique as it is based on a laser cavity. The BER at $20 \mathrm{~Gb} / \mathrm{s}$ is currently limited by the disc's output power and the receivers sensitivity (see eye pattern in Fig. 5). By using recently reported [9] improved silicon couplers with $<2 \mathrm{~dB}$ loss, error free operation at $20 \mathrm{~Gb} / \mathrm{s}$ is well within reach. Numerical simulations have suggested that by driving the MDLs at 20-30 times threshold (as shown in this demonstration), error-free operation at $40 \mathrm{~Gb} / \mathrm{s}$ is also possible. The prospect of integrating hundreds or thousands of these MDL based converters may open the way to elaborate network on a chip designs in the future.

\section{Acknowledgements}

This work was supported by the EU-funded FP7 ICT Project - HISTORIC.

\section{References}

[1] S.L. Danielsen et al, JLT (16), 12, p.2095, 1998

[2] A.C. Turner et al, Optics Exp. (16),7,p.4881, 2008

[3] Q. Xu et al, Opt. Lett.(30), 20, p.2733, 2005

[4] S. Nakamura et al, proc. of OFC 2004, FD3

[5] L. Yong et al,JLT(25), 1, p.103, 2007

[6] O. Raz et al, proc. of ECOC 2009, 4.2.3

[7] T. Spuesens et al, proc. of Group Four Photonics 2009, FA3

[8] M.J.Adams et al, IEEE Proc.(129), 6, p.271, 1982

[9] G. Roelkens et al, APL Vol. 92, No.13, 2008 